\title{
On the Risk of False Positive Identification Using Multiple Ion Monitoring in Qualitative Mass Spectrometry: Large-Scale Intercomparisons with a Comprehensive Mass Spectral Library
}

\author{
Stephen E. Stein \\ National Institute of Standards and Technology, Gaithersburg, Maryland, USA \\ David N. Heller \\ FDA Center for Veterinary Medicine, Laurel, Maryland, USA
}

\begin{abstract}
Analysts involved in qualitative mass spectrometry have long debated the minimum data requirements for demonstrating that signals from an unknown sample are identical to those from a known compound. Often this process is carried out by comparing a few selected ions acquired by multiple ion monitoring (MIM), with due allowance for expected variability in response. In a few past experiments with electron-ionization mass spectrometry (EI-MS), the number of ions selected and the allowable variability in relative abundance were tested by comparing one spectrum against a library of mass spectra, where library spectra served to represent potential false positive signals in an analysis. We extended these experiments by carrying out large-scale intercomparisons between thousands of spectra and a library of one hundred thousand EI mass spectra. The results were analyzed to gain insights into the identification confidence associated with various numbers of selected ions. A new parameter was investigated for the first time, to take into account that a library spectrum with a different base peak than the search spectrum may still cause a false positive identification. The influence of peak correlation among the specific ions in all the library mass spectra was also studied. Our computations showed that (1) false positive identifications can result from similar compounds, or low-abundance peaks in unrelated compounds if the method calls for detection at very low levels; (2) a MIM method's identification confidence improves in a roughly continuous manner as more ions are monitored, about one order of magnitude for each additional ion selected; (3) full scan spectra still represent the best alternative, if instrument sensitivity is adequate. The use of large scale intercomparisons with a comprehensive library is the only way to provide direct evidence in support of these conclusions, which otherwise depend on the judgment and experience of individual analysts. There are implications for residue chemists who would rely on standardized confirmation criteria to assess the validity of a given confirmatory method. For example, standardized confirmation criteria should not be used in the absence of interference testing and rational selection of diagnostic ions. (J Am Soc Mass Spectrom 2006, 17, 823-835) (C) 2006 American Society for Mass Spectrometry
\end{abstract}

$I^{1}$ n qualitative mass spectrometry, mass spectra are used for the identification of specific chemical compounds, just as fingerprints are used to identify individuals. The classical method for identification of a volatile organic compound uses separation by gas chromatography (GC) followed by acquisition of the complete electron-ionization (EI) mass spectrum. Chemical identity is established by comparing the measured retention time and mass spectrum against reference data. This can involve the examination of a large,

Published online April 17, 2006

Address reprint requests to Dr. S. E. Stein, NIST Mass Spectrometry Data Center, A111/221, Gaithersburg, MD 20899-8380, USA. E-mail: steve.stein@ nist.gov comprehensive library of mass spectra [1]. Algorithms used for this examination generally return match factor values that provide an overall measure of spectral similarity, which is assumed to correlate with the likelihood that a reference compound generated the measured spectrum [2]. In the most confident identifications, the only spectrum that matches the measured spectrum is that of the identified compound. It is also important to assess the possibility of a false positive identification, i.e., the chance that the measured spectrum arose from a different compound than the bestmatching reference compound [3].

Because of its sensitivity and selectivity, mass spectrometry has been the method of choice for over $25 \mathrm{y}$ for a wide range of qualitative applications, e.g., detection 
of residues of concern in food. Some residues are bioactive compounds, the ingestion of which may be associated with human health risks. Given the consequences of finding an unhealthful residue, method performance requirements are fairly strict. Confirmation by mass spectrometry refers to the demonstration that an unknown is identical to bona fide standard. Their diagnostic signals must match within some tolerances or windows-known as confirmation criteriathat derive from both instrument variability and method performance requirements. A confirmatory method has a certain identification confidence associated with it, which can also be described as selectivity $[4,5]$. Selectivity is a matter of degree, and might be understood as corresponding to the predicted false positive rate. These measures of qualitative confidence are very slippery concepts to describe in practice.

\section{Background}

The seminal paper on confirmation by mass spectrometry was published in 1978 by James Sphon of the US Food and Drug Administration [6]. EI-MS was the dominant technique in that era, and full-scan EI-MS still provides the most diagnostic and reproducible mass spectral identifications. However, confirming the presence of certain drug residues in food was commonly carried out by monitoring only a few diagnostic ions, mainly to achieve low detection limits. This is the method of multiple ion monitoring (MIM). Sphon recommended that stricter requirements should be applied because MIM data are very limited compared to fullscan methods; most of the mass spectral information isn't collected. Ion ratio matching was invoked for this purpose, i.e., comparison of the relative abundances of ions in normalized mass spectra (along with retention times from online chromatography) against the same parameters in standards to raise the identification confidence of MIM closer to that of full-scan EI-MS.

Sphon presented a case study in which all compounds other than diethylstilbestrol (DES) were eliminated from a contemporary database of over 30,000 EI mass spectra (provided by the National Bureau of Standards, NBS, now NIST, the National Institute of Standards and Technology). There were no false positive identifications when three MIM ions were selected from the DES mass spectrum, with acceptance limits applied to their relative abundance (shown graphically in Figure 1a). This example eventually led to the generally-accepted principle that at least three diagnostic ions were required for mass spectral confirmation. The same test was carried out by Matusik et al. in 1997 using a newer and much larger NIST database, and yielding the same outcome [7]. Also, the same technique was tested using malathion in 2001 (shown graphically in Figure 1b) [8].

In all these cases, the selected ions and their relative abundances were used to eliminate all other library entries, leaving only the search compound as the iden- tified compound. We refer to this technique as delimited ion/abundance database searching, or DIADS. The present study carries out these DIADS experiments on a large scale, by comparing a subset of the NIST EI-MS database against the entire database and looking for trends in the overall results. We refer to the search spectrum, which is compared against library spectra.

There is one key difference between our experiment and those reported by Sphon, Matusik, and Webb. For example, if four diagnostic ions are selected from the spectrum of a search compound, these would consist of the base peak (normalized to $100 \%$ ) and three others at, say, $45 \%, 40 \%$, and $20 \%$ relative abundance (Figure $1 \mathrm{c}$ ). A library spectrum may contain those same ions, but with none of them the base peak in the spectrum (Figure 1d). The library compound might have those fours ions at $50 \%, 22 \%, 20 \%$, and $10 \%$ abundance relative to the base peak. However, in a MIM experiment the data from this compound's base peak is not captured. Hence, the library compound could mimic the search compound in MIM mode, leading to a false positive identification (Figure 1e). This possibility has not been considered before. Essentially, the matching experiments of Sphon, Matusik, and Webb assumed that one of the ions from the search spectrum was the base peak in the library spectrum. This assumption is not necessarily valid, and could have prejudiced those experiments in favor of a unique identification. Our work addresses this problem by introducing another variable for controlling which library spectra are included in each library search. We named this variable the base peak matching abundance, or BMA. The BMA value is the relative abundance of the search spectrum's base peak in the library spectrum, expressed as a fraction. In Figure $1 \mathrm{~d}-\mathrm{f}$, the BMA is $1 / 2$. Clearly, the search spectrum (Figure 1c) and the library spectrum (Figure 1d) are not the same. However, if the minimum BMA for a given search is $\leq 1 / 2$, this library spectrum will be included and a false positive will result. (for details see the Method section below).

\section{The Need for Guidance}

Mass spectral techniques have now proliferated to include atmospheric pressure ionization and tandem mass spectrometry (to name only two of many new techniques). However, large databases of API-LC/MS or MS/MS spectra have not been developed, in contrast to EI-MS [9]. The reasons include the greater degree of variability among MS/MS instruments, and the lower chromatographic resolution in typical LC, although researchers are working to overcome these challenges [10, 11].

Given the range of options now available, it was important to the FDA Center for Veterinary Medicine (CVM) to develop consensus criteria for mass spectral methods, because these guidelines provide direction for drug residue method development. By the mid-1990s, CVM had evolved criteria for the confirmation of drug 

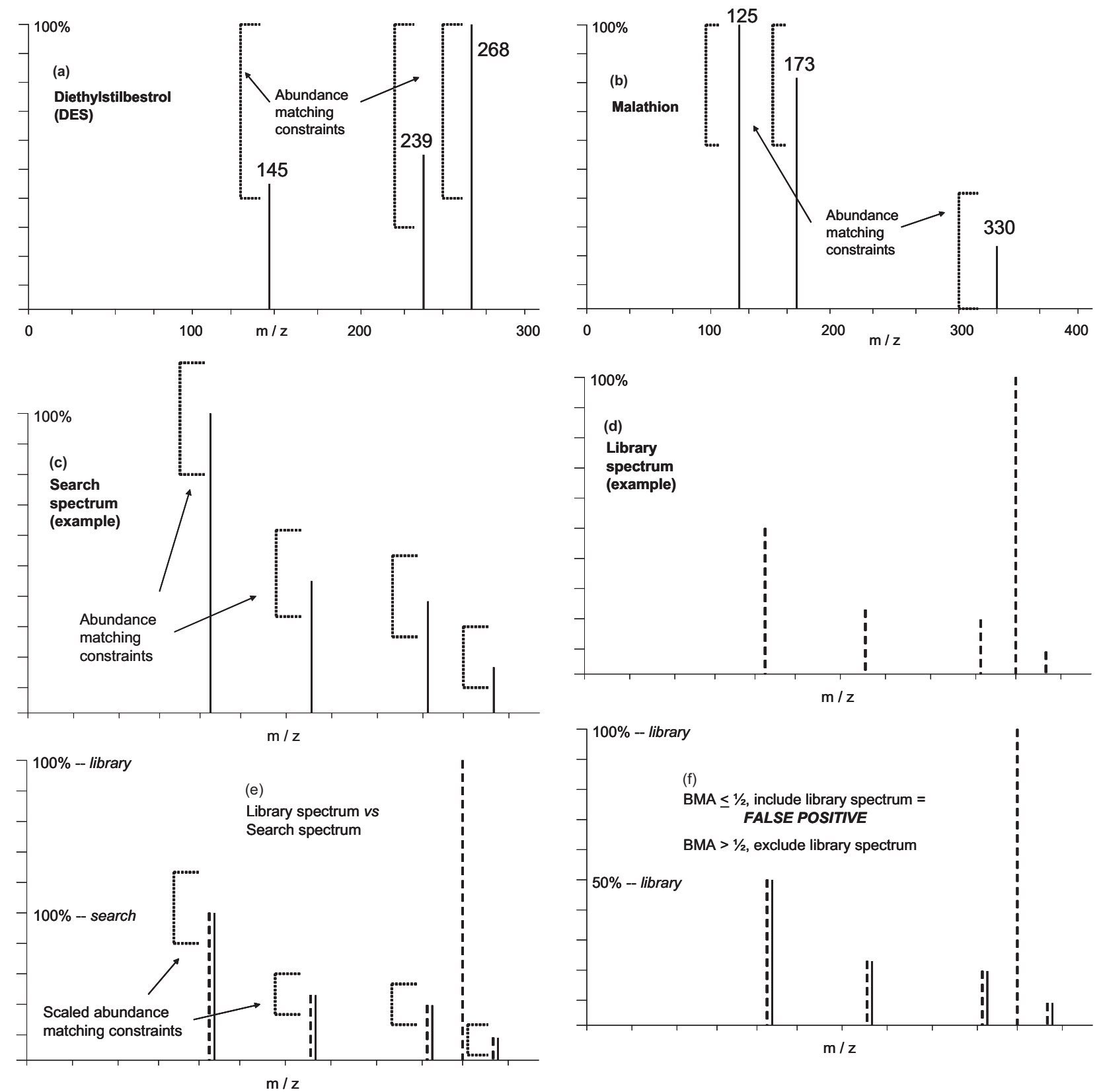

Figure 1. (a) MIM spectrum of diethylstilbestrol, with matching constraints that yielded no false positives in the NIST database [6]. (b) MIM spectrum of malathion, with matching constraints that yielded no false positives in the NIST database [8]. (c) Hypothetical MIM search spectrum, with calculated matching constraints. (d) Hypothetical non-matching MIM library spectrum. (e) Overlay of nonmatching spectra, if search spectrum is scaled to the abundance of the search spectrum base peak in the library spectrum. Matching constraints are scaled accordingly. (f) If BMA $\leq 1 / 2$, then the library spectrum is allowed, leading to a false positive.

residues in animal tissues when human consumption of such residues poses a health risk [12]. A similar process of criteria development led to European data requirements, which are applicable to residues of health concern in food products [13]. The underlying principle in the EU approach is that method selectivity is cumulative. Each piece of chromatographic and mass spectral data can be combined to provide as high a degree of selectivity as the application requires. For example, more diagnostic data points are required to identify a banned substance than for an approved substance whose maximum residue level has been exceeded.

There is a dearth of hard data to support the use of standardized confirmation criteria with MIM acquisition. Guidance documents tend to reflect judgment and consensus among experienced residue chemists, rather than experimental data. Still, these issues are of great significance in regulatory and health applications. Is the 
MIM approach scientifically reasonable compared to full scan analysis? How many diagnostic ions are required for highly confident identifications? How should those ions be selected? How can the risk of false positive identifications using MIM be assessed? How can it be minimized? While the present work does not provide direct answers to these questions, it provides information that may be helpful in answering them. Moreover, results presented here may be of more general interest in understanding factors influencing library search performance. This assessment of selectivity (or identification confidence) also addresses the recommendations of a recent committee report [14], which was concerned with establishing the fitness for purpose of mass spectrometric methods.

\section{Materials and Methods}

The 2002 version of the NIST/EPA/NIH Mass Spectral Library [15] provided the spectra for these studies. All calculations were performed on a personal computer using ' $C$ ' code derived from the NIST/EPA/NIH Mass Spectral Library and Search Program [16]. To simplify computations with the DIADS experiment, ion abundances were normalized to 1 , rather than $100 \%$.

Only those spectra that satisfied the following requirements were used:

1. contained peaks $<0.2 \%$ relative abundance (abundance $<0.002$ );

2. contained at least 10 peaks;

3. molecular weight $<1000 \mathrm{u}$;

4. not a stereoisomer of another spectrum (when multiple stereoisomers or $\mathrm{Z} / \mathrm{E}$ isomers were represented, only the first spectrum in the library was used).

These restrictions reduced the number of potential library spectra for this study from 147,198 to 96,464 . The minimum BMA value was used as an additional selection criterion. As stated above, BMA is the abundance of a peak in a library spectrum at the $\mathrm{m} / \mathrm{z}$ of the base peak in the search spectrum, expressed as a fraction. For instance, if the base peak of the search spectrum is $\mathrm{m} / \mathrm{z}$ 91 , and the library spectrum has a peak at $m / z 91$, which is 0.25 relative to the library spectrum's base peak, then BMA is $1 / 4$. The library selection criteria included a minimum BMA value that ranged from 1 down to $1 / 128$, diminishing by $1 / 2$ for successive values. If the library spectrum's BMA was more than the minimum BMA defined in the current search constraints, it was included in the search. See Table 1 for examples. The \#FP was determined at eight different minimum BMA levels. The values reported in this work represent spectra with BMA values at or above the specified minimum BMA, not just at a specified minimum BMA.

Each search determined the number of selected library spectra that matched the search spectrum according to certain peak constraints. The search spectrum was eliminated from the library before performing a search, so all library spectra that matched the search spectrum for a given set of constraints were false positives. For a given search, the number of false positive spectra (\#FP) divided by the total number of selected library spectra considered in that search gave the false positive probability (FPP). Every tenth spectrum in the reduced library was used as a search spectrum, meaning that roughly 9600 search spectra were used.

For a set of constraints to give a unique identification, there could be no matching spectra (i.e., $\# \mathrm{FP}=$ FPP $=0$ ). The constraints were:

Number of peaks (NP). The number of different $\mathrm{m} / \mathrm{z}$ values (peaks) used for matching. NP ranged from $1-8$. The NP most abundant peaks in the search spectrum were selected.

Abundance window $(A W)$. This constraint sets upper and lower bounds on the difference in abundance between search and library spectra at a given $\mathrm{m} / \mathrm{z}$ which is considered a match. In this work AW is assumed to be proportional to the square root of the relative abundance of peaks in the search spectrum. The AW constraint does not apply to the base peak of the search spectrum since for that peak matching is determined by the minimum BMA value alone (a matching library peak must have an abundance greater than BMA).

This square root dependence on abundance approximately expresses the variations allowed in previous work modeling the variability of ion abundances [17]. It enables the consistent application of abundance matching criteria. A key qualitative trend often used in setting matching criteria is expressed by the square root dependence, namely that as abundance diminishes, the matching tolerance narrows in terms of abundance measured relative to the base peak (absolute value), but expands as a fraction of the abundance of the peak itself (relative value). This scheme results in matching windows that are slightly wider than those set by two guidance documents (compare Figure $2 \mathrm{a}$ and $\mathrm{b}$ ).

In this work, the abundance window, $\mathrm{AW}$, is presumed to increase with decreasing abundance. The entire window could be made larger or smaller to test the effect on FPP, by applying a weighting factor, the abundance window multipler (AWM). Initial studies were carried out with AWM of 0.25 , as follows:

$$
\mathrm{AW}=1+\mathrm{AWM}^{*} \operatorname{sqrt}[\mathrm{S}(\mathrm{ml}) / \mathrm{S}(\mathrm{m} n)] .
$$

where $S(\mathrm{~m} 1)$ and $S(m n)$ are relative abundances of the first and $n t h$ largest peaks in a given spectrum (see later)

The resulting upper and lower bounds are plotted as a function of abundance in Figure 2. If the lower bound is less than the smallest peak in a library spectrum, then that spectrum is ignored, in effect, reducing the size of library for that search. This strategy thus ignores library 
(a) Abundance matching bounds for various peak abundances $(A W M=0.25)$

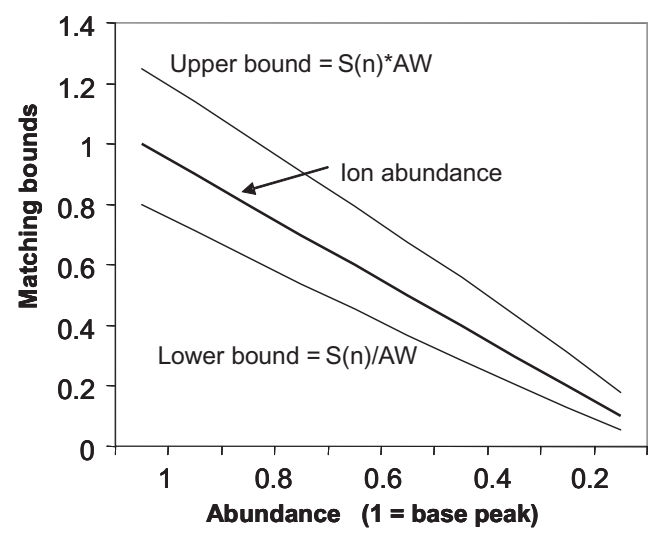

(b) Abundance matching bounds From other sources

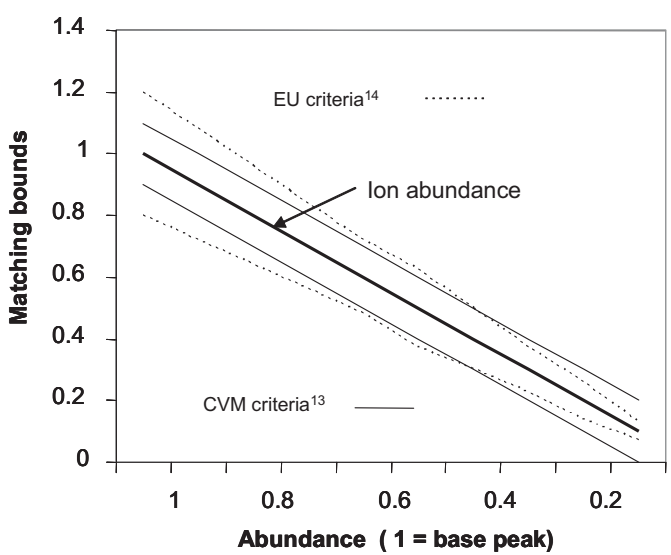

Figure 2. (a) Schematic showing the upper and lower abundance matching bounds resulting from an AW value of 0.25 , for ions with abundance 1-0.1 (heavy solid line). (b) Schematic showing the upper and lower abundance matching bounds described by the EU [14] (light dashed line) and by CVM [13] (light solid line).

spectra that are lacking matching small peaks because they were not measured with sufficient sensitivity.

The use of BMA and AW constraints are illustrated as follows: Let $\mathrm{m} / \mathrm{z}$ values in the search spectrum, ordered by abundance, be represented as: $\mathrm{m} 1, \mathrm{~m} 2, \ldots$ $\mathrm{m} n$... and their relative abundances as: $\mathrm{S}(\mathrm{m} 1), \mathrm{S}(\mathrm{m} 2)$, .. $\mathrm{S}(\mathrm{m} n), \ldots$ Note that $\mathrm{m} 1$ is the base peak in the search spectrum and $\mathrm{S}(\mathrm{m} 1)=1$. Let $\mathrm{L}(\mathrm{m} 1), \mathrm{L}(\mathrm{m} 2), \ldots$ represent the abundances of peaks in the library spectrum for the same set of $m / z$ values. The BMA value is $\mathrm{L}(\mathrm{m} 1) / \mathrm{S}(\mathrm{m} 1)$. For any other peak $\mathrm{m} n$ to match, the following must hold:

$$
\frac{1}{\mathrm{AW}} * \mathrm{~S}(\mathrm{~m} n)<\frac{\mathrm{L}(\mathrm{m} n)}{\mathrm{L}(\mathrm{m} 1)}<\mathrm{AW} * \mathrm{~S}(\mathrm{~m} n)
$$

where $A W$ is the abundance window. If this expression is true for all NP peaks in the library spectrum, there is a match (i.e., false positive).

\section{Results and Discussion}

\section{Presentation of Results}

For a given set of constraints, the set of all search spectra produced many thousands of FPP values. These results were simplified for presentation by determining the median FPP value of results from each set of constraints (Figure 3). Median values were used instead of average FPPs, because they represent results of typical searches. Averages (or means) exaggerate the effect of the relatively small numbers of searches that yield exceptionally large FPP values.

Some search spectra produced fewer false positives than others. These were relatively selective spectra, that is, their overall diagnostic value was greater than that of most other spectra for a given set of constraints. For example, a relatively selective spectrum might have an FPP value less than $90 \%$ of the other search spectra, and thus would be in the 90th FPP percentile-these spectra would have a much lower risk of producing false positives. We used these FPP percentiles to examine this selectivity feature more closely, and to explore the reasons why some spectra were more or less selective.

\section{Medians and Distributions of False Positive Probabilities}

In Figure 3, median FPP values are plotted over a range of numbers of peaks, NP, at minimum BMA values diminishing by halves from 1 to $1 / 128$. These median FPP values decline by roughly a factor of 10 for each additional peak (i.e., as NP increases by 1). Clearly, increasing the number of peaks does reduce the false positive probability. Also in Figure 3, the median FPP values increase as minimum BMA values decrease, because there is an increasing chance of false matches when smaller peaks are allowed. The selected minimum BMA value allows library spectra having peaks at the $\mathrm{m} / \mathrm{z}$ of the base peak in the search spectrum that are higher than this value. Decreasing the minimum BMA value is functionally equivalent to increasing the sensitivity of MIM experiments (looking deeper into the "grass"). On the other hand, a minimum BMA of 1 means that the base peak in the search and library spectrum occur at the same $\mathrm{m} / \mathrm{z}$. Note that the FPP becomes increasingly less sensitive to the minimum BMA value as the minimum BMA values decline; the plot lines nearly merge at the smallest values.

Individual FPP values depend on the spectrum from which the peak constraints were derived. This depen- 


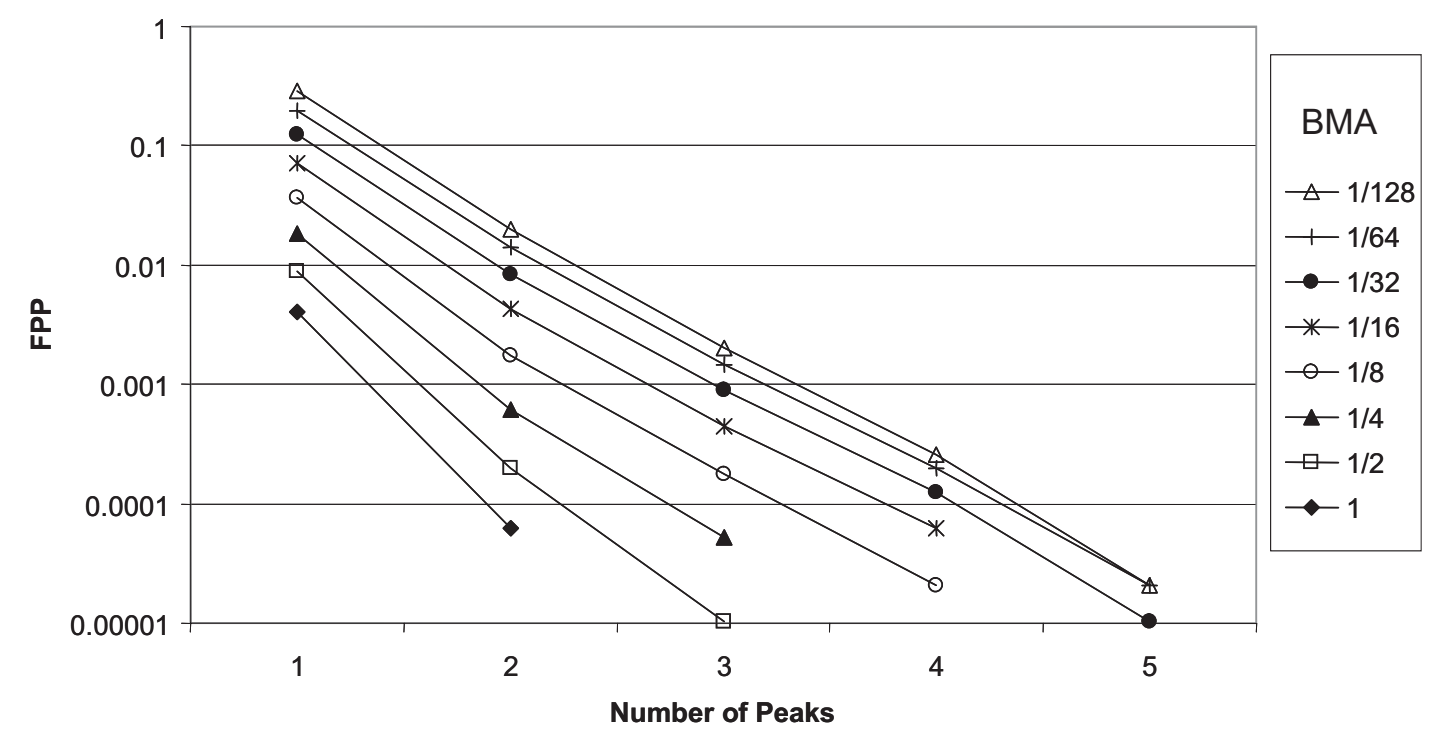

Figure 3. Median false positive probabilities (FPP) as a function of the number of peaks selected (NP), plotted at eight values of minimum BMA.

dence is illustrated in the distributions of FFP values shown in Figure 4. a, b and c, which correspond to NP values of 2,3, and 4, respectively, over the range of minimum BMA values from 1 to $1 / 128$. These distributions are represented by the median FPP of each percentile of observed FPP levels, which as discussed above, are measures of selectivity.

Figure $4 a-c$ "spread out" the results shown in Figure 3. Note that the overall median FPP values which are plotted in Figure 3, represent the 50th percentile values in Figure $4 \mathrm{a}-\mathrm{c}$ (where the dashed line intersects each curve). With the FPP results shown as curves rather than single points, it is still quite evident that FPP decreases steadily as NP increases. Furthermore, the shapes of these curves contain more information about variations in spectral selectivity.

The slopes of the curves in Figure $4 \mathrm{a}-\mathrm{c}$ decline with declining minimum BMA. This indicates that spectrumto-spectrum variations in FPP decline with decreasing minimum BMA. FPP values for the most selective spectra, that is, those with the smallest FPP and highest percentile ranking, increase more rapidly with declining minimum BMA than do values for less selective spectra. For instance, for $\mathrm{NP}=3$, reducing the minimum BMA from 1/4 to $1 / 64$ increases FPP by a factor of 51 for a set of constraints in the 75th percentile, by a factor of 23 at the 50th percentile (median), and a factor of only 11 at the 25 th percentile. The key point here is that the more selective the spectrum, the more the risk of false positive identification increases with decreasing minimum BMA (or, by inference, increasing instrument sensitivity).

Another important feature of Figure $4 a-c$ (and Figure 3 ) is the reduced separation of the curves with diminishing BMA, apparently converging to an asymptote. This limit reflects the case of very high sensitivity where peaks of finite abundance are ex- pected to be found at almost all $\mathrm{m} / \mathrm{z}$ values below the molecular weight of the compound. At this limit there would be no further influence of declining minimum BMA (or increasing instrument sensitivity) on FPP.

Three characteristic features of each of the curves in Figure $4 \mathrm{a}-\mathrm{c}$ may be distinguished. One is the stepped behavior and abrupt termination of the curves at low FPP. This is a simply a consequence of the fact that false positive results are measured as integers (numbers of matching spectra) and at a sufficiently high selectivity, no false positives are found (FPP is zero). For instance, at minimum BMA $=1$ for $\mathrm{NP}=3,55 \%$ of search spectra show no false positive results, so FPP values at percentiles $>45$ cannot be reported. Another feature is the increased slope magnitude at the highest FPP percentile (near the 100th percentile). In this region, the number of false positive results approach zero in a linear fashion. For example, there are approximately twice as many false positive results at the 98 percentile compared to the 99 percentile. This appears as downward curvature on the $\log$ axis used in these figures.

A third distinctive feature is seen in the lowest FPP percentile region, where a rapid increase in FPP occurs as the FPP percentile approaches zero. This originates from the large subset of spectra that have the same major fragment peaks. Examination of these results shows that these nonselective spectra contain major peaks corresponding to the most common hydrocarbon fragment ions.

\section{Abundance Windows, AW}

Ideally, abundance windows should be set just wide enough to accept normal variations in the abundances of peaks in the target compound. Settings 
(a) FPP Distributions, NP=2

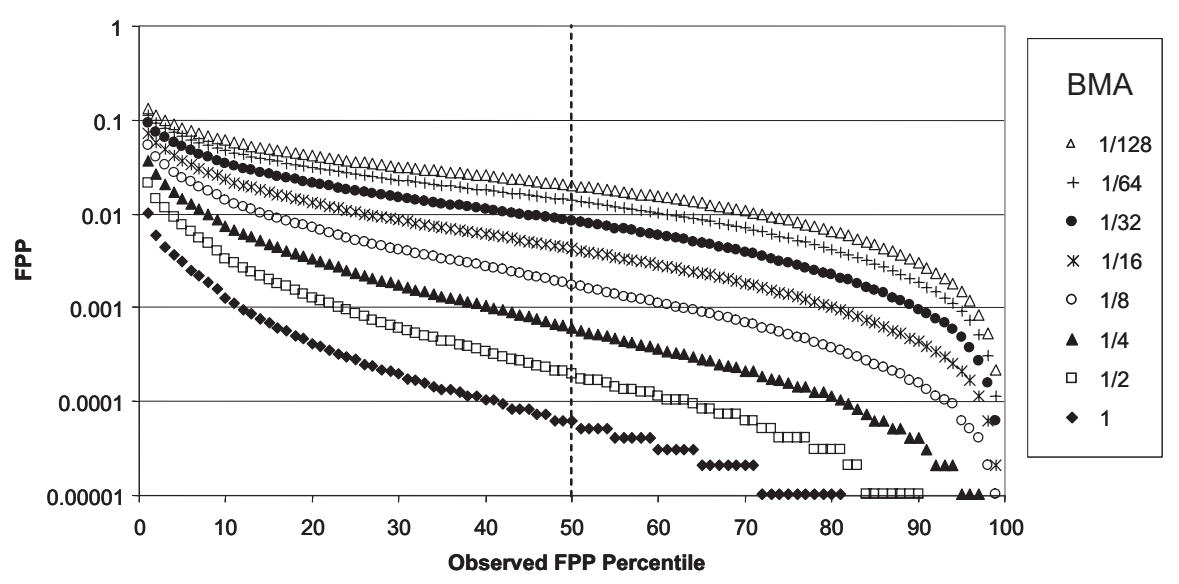

(b) FPP Distributions, NP=3

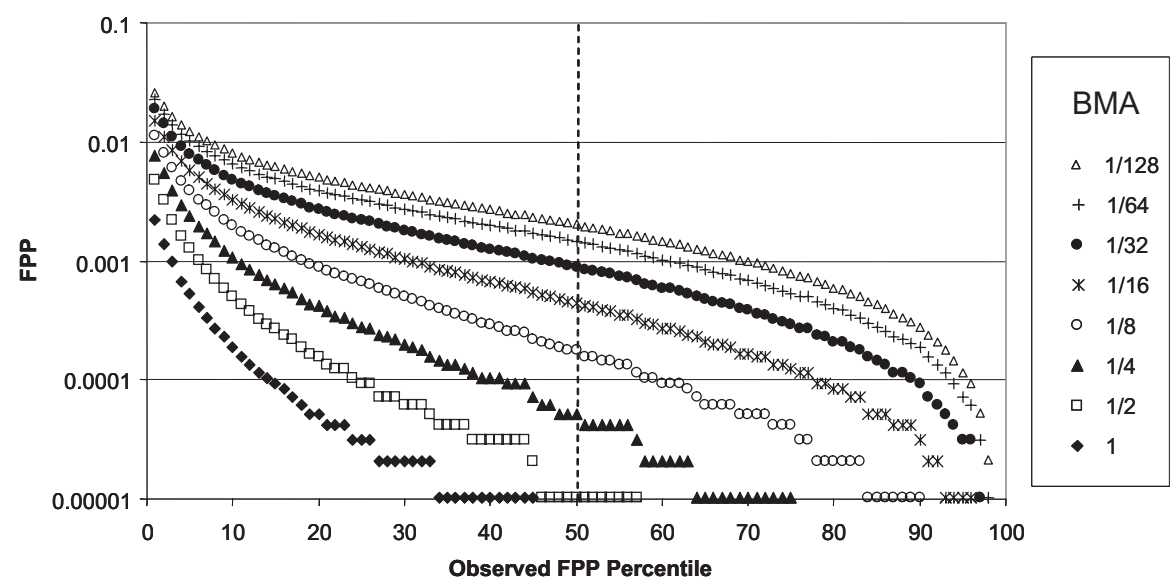

(c) FPP Distributions, NP=4

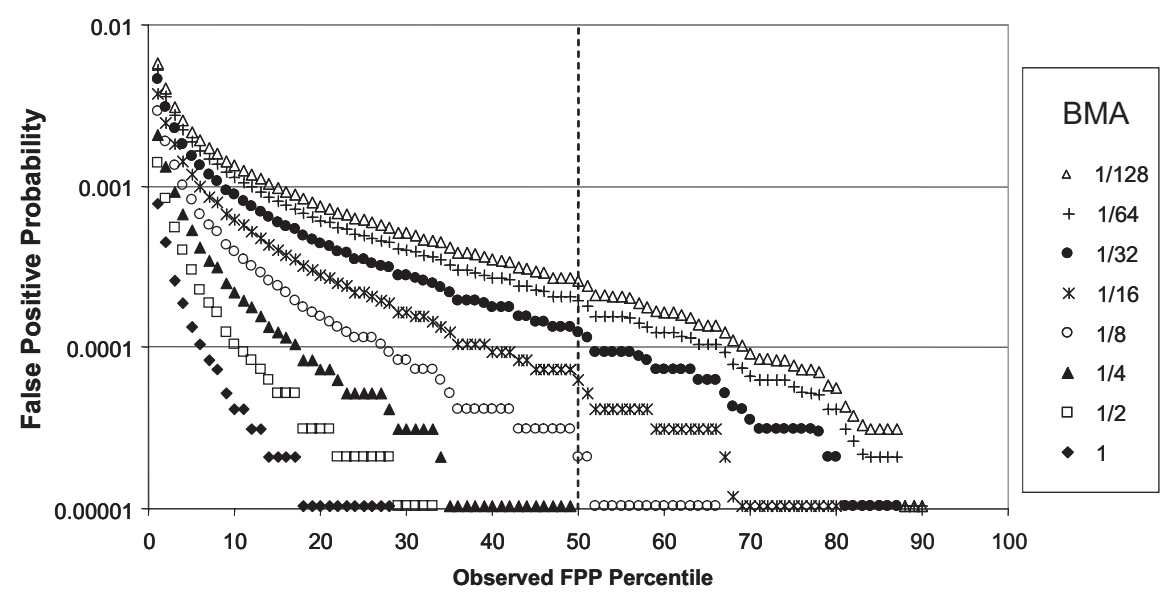

Figure 4. (a) False positive probabilities (FPP) when $\mathrm{NP}=2$, at eight values of minimum BMA, as a function of observed FPP percentile. (b) False positive probabilities (FPP) when NP = 3, at eight values of minimum BMA, as a function of observed FPP percentile. (c) False positive probabilities (FPP) when NP $=4$, at eight values of minimum BMA, as a function of observed FPP percentile.

wider than necessary will needlessly increase the risk of false positive identification. The overall effect of changes in abundance windows on FPP is roughly predictable. For sufficiently small abundance windows, numbers of false positive identifications should be simply proportional to the width of each 


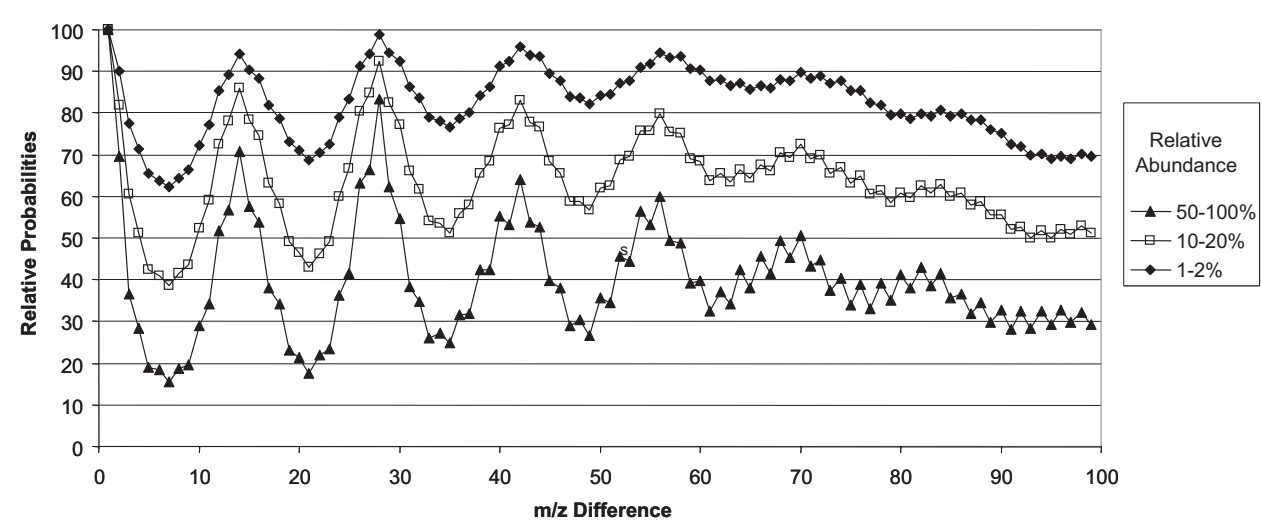

Figure 5. The relative probability of two peaks occurring in the same spectrum, plotted as a function of the mass difference and the relative abundance of the peaks.

abundance window. This was verified for the case where the Abundance Window Multiplier AWM was changed from 0.25 to 0.15 , where resulting test median numbers of false positive results diminished by $(0.25 / 0.15)^{\mathrm{NP}-1}$.

In Figure 2, an Abundance Window Multiplier of 0.25 is somewhat wider than EI matching tolerances recommended by CVM [13] or by the EU [14]. An abundance window multiplier of 0.15 narrows the bounds to be roughly midway between those of CVM and the EU throughout the range of Figure $2 \mathrm{~b}$ (not shown).

\section{Peak Occurrence Probabilities and Correlation}

The probability of finding a single library peak at a given $\mathrm{m} / \mathrm{z}$ and within a specified abundance range may be derived simply from an analysis of all peaks in the library. These can be viewed as independent peak occurrence probabilities. Related quantities have been used as weighting factors in library searching [18]. However, probabilities of finding more than one peak in a spectrum are far more complex. This is illustrated in Figure 5, where probabilities of finding pairs of peaks within fixed abundance ranges are plotted as a function of their differences in $\mathrm{m} / \mathrm{z}$ values. Note that the correlation is significantly greater for larger than for smaller peaks and follows well-known "mod 14" cycles, originating primarily from differing numbers of methylene units in ion fragments. Peak separation by 1 or $2 \mathrm{~m} / \mathrm{z}$ is also common due to different degrees of $\mathrm{H}$-loss during fragmentation, $\mathrm{O} / \mathrm{CH}_{2}$ differences and isotopes.

The greatest difference between these co-occurrence probabilities is for $\mathrm{m} / \mathrm{z}$ separations of 1 and 7 , where probabilities differ by a factor of 6.7 for large peaks. This diminishes to a factor of only 1.6 for peaks between 1 and $2 \%$ of the base peak. The variations in Figure 5 are, in effect, averages of even more complex behavior, where occurrence probabilities depend on the specific $\mathrm{m} / \mathrm{z}$ and abundance values of other peaks in the spectrum. In effect, the occurrence probability at each $\mathrm{m} / \mathrm{z}$ in a spectrum depends on all of the other peaks. "Neutral losses" provide another source of correlation, where $\mathrm{m} / \mathrm{z}$ is measured relative to the molecular ion. For example, $37 \%$ of spectra have a peak with abundance above $1 \%$ of the base peak for the neutral loss of methyl from the molecular ion. All of these correlations originate from the "non-random" nature of mass spectra, which of course reflects library composition and dissociation rates. Peak correlation is clearly a dominant feature in mass spectra. A truly random selection of just a few peaks with typical abundance windows rarely match spectra even in very large spectral collections.

It was possible to estimate FPP in the absence of peak correlation. First, individual peak occurrence statistics were compiled by tallying the number of times each mass peak appeared in the entire database. The product of these probabilities for each peak falling within a prescribed abundance range provides a measure of uncorrelated FPP values, which are those expected in the absence of peak correlation. The uncorrelated FPP values were compared to the measured FPP values corresponding to each set of constraints. Figure $6 a, b$, and c. show results for $\mathrm{NP}=3$ at $\mathrm{BMA}=1,1 / 8$ and $1 / 64$ (respectively) as a function of observed FPP percentile. They show actual measured median FPP values as well as uncorrelated FPP median, first and third quartile values (i.e., 25th and 75th percentiles). Each of these values was derived from the spectra falling within a $10 \%$ percentile window centered at corresponding FPP percentile values.

While actual FPP values follow uncorrelated FPP trends, actual FPP values are significantly larger, reflecting positive correlation. At BMA $=1$, observed values are higher by a factor of 12. At lower BMA values this ratio decreases to roughly a factor of 2 , reflecting the lower degree of correlation for the smaller peaks (Figure 5). Also, at low BMA values this ratio increases by a factor of about 2 from low to high selectivity.

Spectrum-to-spectrum FPP variations are substantial, as shown by the large differences between first and third quartile median uncorrelated values $(1 / 2$ of all values falls between the first and third quartile). Note that this wide range of values are for searches that have 
(a) Actual \& Uncorrelated FPP NP $=3$, BMA=1
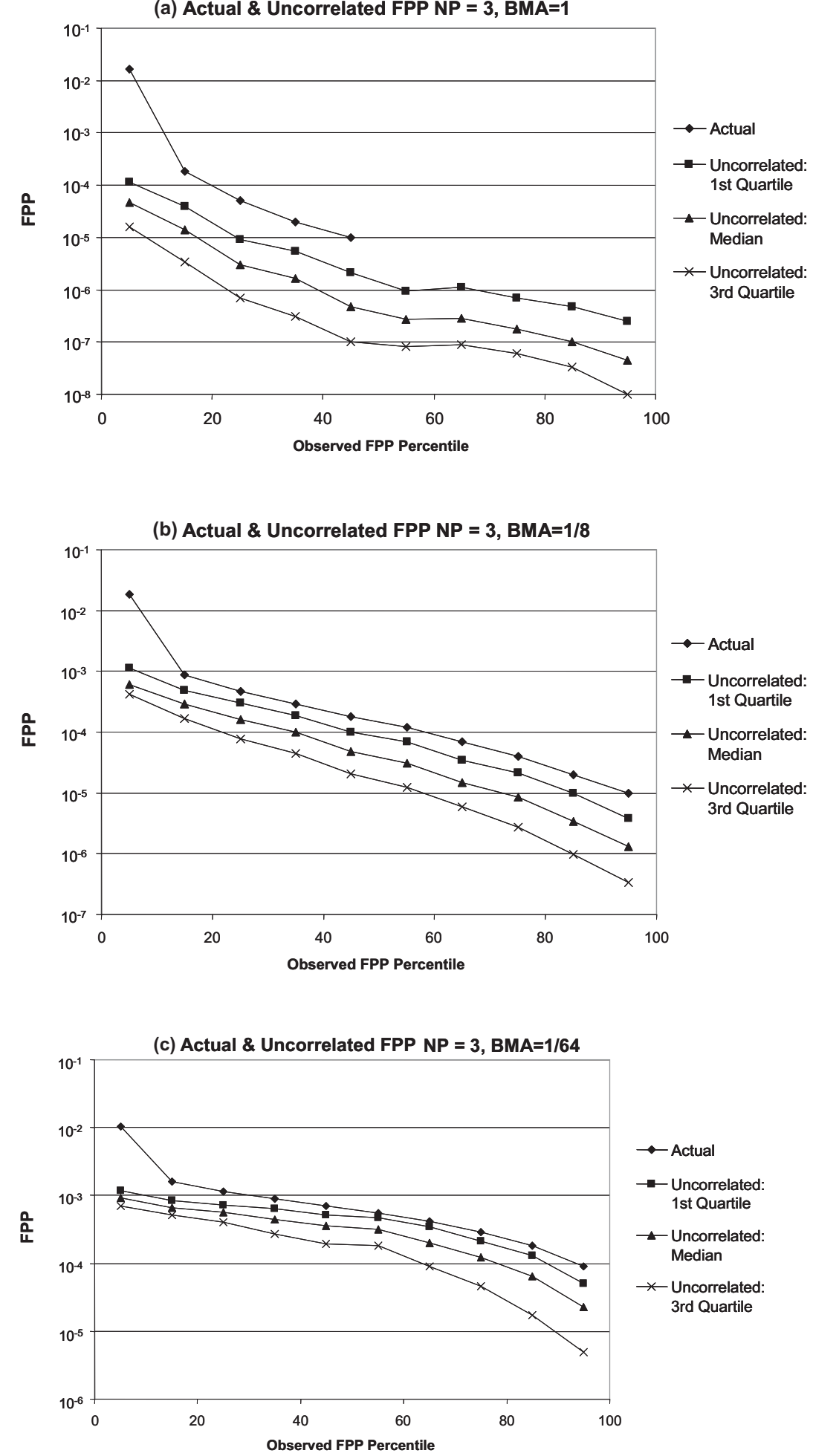

Figure 6. Actual and computed uncorrelated false positive probabilities for $\mathrm{NP}=3$ at minimum $\mathrm{BMA}=1(\mathbf{a}), 1 / 8(\mathbf{b})$, and 1/64 (c), as a function of observed FPP percentile.

a narrow range of actual FPP values. At BMA $=1$, the first and third quartile values differ by a factor of 12 , coincidentally the same as the difference between observed and uncorrelated median values. This dispersion changes little over the range of selectivity. At lower
BMA values, this dispersion values is significantly narrower for lower selectivity spectra and increases to about 12 , the same value found for $\mathrm{BMA}=1$, for the most selective spectra.

To summarize, peak correlation increases false pos- 


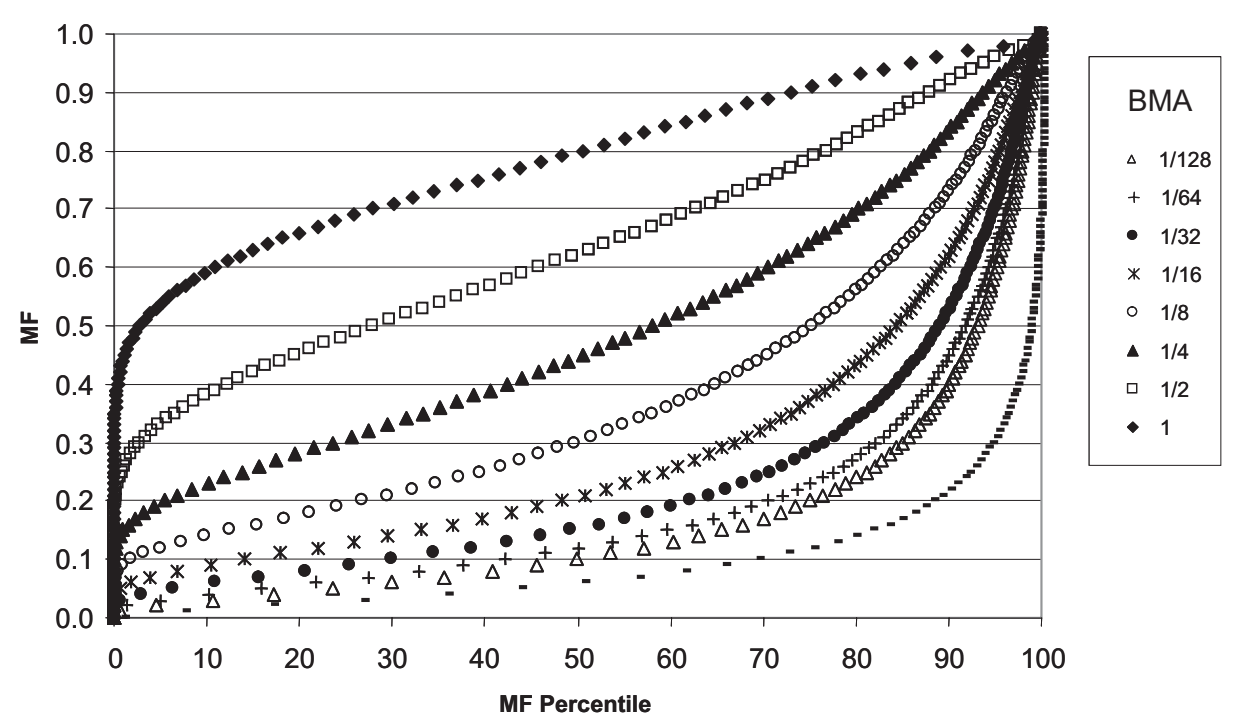

Figure 7. The distribution of match factors for full search and library spectral pairs that produce false positive results in the present MIM studies for NP $=3$, over a range of minimum BMA values. The top curve depicts results for $\mathrm{BMA}=1$ and each successive lower curve decreases minimum BMA by a factor of two, except for the bottom curve, which shows the match factor distribution for random pairs of spectra.

itive probabilities for $\mathrm{NP}=3$ by about an order of magnitude for large peaks and a factor of two for small peaks. It is also responsible for significant spectrum-tospectrum fluctuation, which results in a dispersion of values for searches having the same actual FPP values, where first and third quartile values differ by more than a factor of ten.

\section{The Role of Similar Spectra}

Traditional mass spectral library searching finds and ranks the library spectra that most closely resemble the search spectrum. A close match of a complete spectrum, especially if it contains multiple high abundance peaks, almost invariably means that the compound producing the reference spectrum is the same, or closely related, to the compound producing the search spectrum. Since a good overall match generally requires that the major peaks in the two spectra match, these matching spectra are the major contributors to the FPP of the present studies at high BMA values. Therefore, results at high BMA values are expected to depend most strongly on the specific compounds represented in the library. If, for instance, a number of positional isomers (the dimethylbenzenes, for example) having equivalent spectra were added to the library that originally contained no such isomers, at minimum BMA $=1 \mathrm{FPP}$ values for them would increase substantially, and the computed selectivity would correspondingly decline.

On the other hand, a match at low minimum BMA will arise largely from accidental matching of peaks in spectra quite different than that of the search spectrum. The abundances of principal peaks in the two spectra cannot match. Therefore, library compounds whose spectra match at low minimum BMA will generally bear little resemblance to the search compound, so that these false positive results will depend little on the composition of the library. Moreover, large numbers of matches are generally found at low minimum BMA values, thereby minimizing statistical fluctuations.

As a measure of the similarity of two full-scan spectra, we use the cosine of the "angle" between two spectra as a measure of overall spectral similarity [2, 19], referred to here as a match factor (MF). Figure 7 shows the distribution of this measure of spectral similarity for the search and library spectral pairs that produce false positive results for $\mathrm{NP}=3$ over a range of minimum BMA values. These distributions are represented as percentiles (percent of spectra with Match Factors above specified values). For comparison, the distribution of similarity values for random pairs of spectra in the library is also shown (bottom curve).

The BMA $=1$ plot shows that the bulk of the library spectra producing false positive identifications for cases where the base peaks match are very similar to the search spectra. The median value (50th percentile) of 0.8 is generally considered to be a good overall match and three-quarters of all identifications have a match factor greater than 0.7 , a value which generally associated with substantial spectral similarity. Match factors decline drastically with declining BMA. At BMA $=1 / 32$, the median value is 0.15 and only $5 \%$ of false positive spectra have match factors above 0.7 . This median value is about double the value for random pairs of spectra.

These results clearly distinguish two extremes of false positive identifications; matches due to similar spectra (most apparent when minimum BMA $>0.5$ ) and matches due to more-or-less random groups of small peaks in a spectrum (lower minimum BMA). The higher BMA matches are those of concern in traditional, full- 
spectrum searching and in earlier considerations of MIM false positive risk, while the lower minimum BMA can dominate when the base peak of the matching spectrum in not known, as in MIM experiments. The former can depend greatly on the specific distribution of compounds in the library (or sample), while the latter will not.

\section{Uniqueness of Individual Spectra}

The above analysis examines medians and distributions of false positive results derived from large numbers of searches. Results for individual spectra will now be examined.

First, we examine diethylstilbestrol (DES), the spectrum used by Sphon [6] to demonstrate the discriminating power of three peaks. His finding in 1978, that no spectrum in the library matched the three principal peaks of DES, still holds today despite the fact that the present library has over four times as many spectra than the one he used. According to Figure 4 a for BMA $>1 / 2$, in terms of selectivity this places that set of peaks in the top half of spectra in the library.

False positive matches for DES were found at lower BMA values, starting at BMA $=1 / 8$. Over the minimum BMA range from $1 / 8$ to $1 / 128$, in steps of two, median numbers of false positive matches were 2, 7, 21, 44 , and 70 (of 96,464 library spectra considered), corresponding to selectivity percentiles of $81,82,80,78$, and 77 (from Figure $4 a$ ). At BMA $=1 / 64$, for example, in terms of the median number of false positive spectra, the three peaks from DES are only about four times as diagnostic as those of the median spectrum in the library.

In a re-examination of Sphon's ideas, Webb used malathion to examine the three-peak criterion. Like DES, no false positive spectra were found for NP $=3$, for BMA $>1 / 8$. Over the same FPP range used for DES, numbers of false positive spectra were: $4,8,27,51$, with corresponding selectivity values of $74,80,76,75$, and 68 . DES and malathion spectra clearly show similar patterns of diagnostic value.

As an example of a spectrum of low diagnostic value consider dodecane, with principal peaks at $m / z 57$ (base peak), $43(78 \%)$, and 71 . (51\%). For NP $=3$, starting from $\mathrm{BMA}=1$ and halving BMA seven times in succession, numbers of matching library spectra were $269,457,611,774,994,1229,1506$, and 1763 . Within $25 \%$, all of these values correspond to spectra at the $1 \%$ level of selectivity ( $99 \%$ of sets of three peaks have smaller FPP values). For $\mathrm{NP}=2$, selectivity percentiles increase with BMA, going from $3 \%$ at BMA $=1$ down to $9 \%$ at $\mathrm{BMA}=1 / 128$. For $\mathrm{NP}=3$, selectivity remains at less than $1 \%$ throughout the range of BMA values.

It is instructive to compare the DES and dodecane results as a function of minimum BMA. Above BMA = $1 / 8$, the top three peaks of DES lead to 600 times fewer false positives than those of dodecane. This ratio diminishes steadily with decreasing BMA to a factor of 25 at
$\mathrm{BMA}=1 / 128$. Not only does the risk of false positives increase substantially with declining BMA, but the effect on the most diagnostic spectra is the most dramatic.

\section{Other Methods of Peak Selection}

In the present studies, the most abundant NP peaks were selected for library-searching. This method may not produce the most diagnostic set of peaks and therefore may not generate the lowest possible uncorrelated (theoretical) FPP values. The correlations between uncorrelated and observed FPP shown in Figure 6 indicate that lower theoretical FPP values will generally result in lower actual FPP values. Peak selection in practice should be based on the most diagnostic set of peaks. One way of identifying the "best" peaks is to determine numbers of false positive results using different possible sets of peaks. Lacking guidance, practicing residue chemists must conduct such experiments in their imagination, based on experience with a variety of compounds. However, the DIADS experiment could be used to test rules-of-thumb concerning peaks selection, such as (in EI-MS) to select high mass ions and avoid nonselective low mass ions, or (in MS/MS) to select product ions that are neither too low in mass nor too close to the precursor ion mass. This may also lead to avoiding peaks differing by 1,2 , or $14 \mathrm{~m} / \mathrm{z}$ units. Of course, many spectra simply do not possess a sufficient number of significant peaks to afford such choices.

\section{Summary and Implications}

This study provides a measure of the increased risk for false positive identification in MIM determinations when increasingly smaller peaks are allowed to match the base peak of the search spectrum (i.e., lower base-peak matching abundance, BMA, requirements). Further, the rate of increase in risk with lower BMA tends to be greater for more selective spectra. This problem is avoided in full spectrum matching. The increase in risk with diminishing BMA approaches a limit at low base-peak matching abundances. For three peaks in a typical spectrum, the median value for numbers of false positive results increases by a factor of 200 from BMA $=1 / 2$ to $1 / 128$, the latter being near the limiting value.

The present studies also show that, in terms of median numbers of false positive values, each added peak typically reduces the false positive risk by about an order of magnitude regardless of the number of peaks or the size of matching peaks in the false positive spectrum. However, there is a significant spectrum-to-spectrum variability that originates from peak correlation, so that FPP values for various sets of peaks may not follow this trend closely. It was found that computed individual peak occurrence probabilities follow median numbers of false positive results. Also, peak correlation significantly increases 
the number of false positives results, typically by a factor of 12 for three-peak matching, and also leads to significant spectrum-to-spectrum variations. Because of the difficulty in expressing these correlations, the only effective way to measure selectivity for a set of peak constraints derived from a given spectrum is to determine the numbers of false positive results that they produce.

While the present results were derived entirely from the analysis of EI spectra, the general conclusions should be applicable to other mass spectral identification where only a few peaks in the spectrum are monitored and base peaks are not known. In MS/MS experiments, for example, so-called multiplereaction monitoring (MRM) determinations typically have such restrictions. Here product ion abundances are typically measured at a single, predetermined $\mathrm{m} / \mathrm{z}$ value for a preselected precursor ion. The ion occurrence statistics is not expected to be radically different from those in EI spectra since both are primarily a product of the dissociation of weak bonds in organic ions.

One potentially serious source of false positive risk is not addressed directly in this work, namely that due to the interference of specific compounds with similar structures and spectra. In the present studies, such interferences will depend on the details of library composition and, while results at $\mathrm{BMA}=1$ describe typical behavior, results for a given spectrum will depend on the compounds possibly present in the specific system under study

This study has implications for the selection of appropriate criteria in qualitative mass spectrometry. The work of Sphon, Matusik, and Webb supported the use of at least three ions for confident identifications, and highlighted the value of full scan methods. Our DIADS experiments showed that only some compounds possessing a few highly diagnostic ions (such as DES and malathion) do not produce false positives when selecting three ions and applying abundance tolerances. However, we found that even highly selective spectra can produce many false positive identifications in DIADS experiments that used only their three largest peaks. Fortunately, our studies also point to steps for further reducing the risk of false positives in MIM methods:

1. consider the diagnostic mass difference between pairs of ions, i.e., avoid highly probable peak correlations such as $14 \mathrm{u}$ differences due to methyl loss or 18 u due to water loss;

2. evaluate full-scan data in the elution range to assess the influence of minor ions from major co-eluting peaks, and test multiple sources of matrix for such endogenous interferences;

3. identify structurally-similar compounds and metabolites for interference testing;

4. Increase the number of ions monitored;

5. Reduce the relative abundance matching tolerance;
6. Examine comprehensive mass spectral libraries for possible false positive identifications.

These considerations apply to the sole use of mass spectrometry for identification purposes. Of course, many additional elements make up a complete regulatory method-extraction, chromatography, validation, quality control, etc. For example, confirmatory data used in legal cases will almost always include on-line chromatography, so a retention time match will add additional confidence to the identification.

This study supports the principle that as more peak constraints are applied, the selectivity of the method increases. However, one thorny problem has emerged: a given set of peak constraints will not have the same selectivity for different compounds. Standardized confirmation criteria do not yield the same false positive probability for different compounds because of the unique structure of each compound and the varying probabilities of similar compounds or mimics. Standarized confirmation criteria should be seen as a starting point for method development, not an endpoint, so specific criteria can be tailored to the stated purpose of the analysis.

\section{References}

1. Auslos, P.; Clifton, C. L.; Lias, W. G.; Mikaya, A. I.; Stein, S. E.; Tchekhovskoi, D. V.; Sparkman, O. D.; Zaikin, V.; Zhu, D. The critical evaluation of a comprehensive mass spectral library. J. Am. Soc. Mass Spectrom. 1999, 10, 287-299.

2. (a) Stein, S. E.; Scott, D. R. Optimization and testing of mass spectral search algorithms for compound identification. J. Am. Soc. Mass Spectrom. 1994, 5, 859-866; (b)Stein, S. E. Probabilities of correct identification from results of MS library searching. J. Am. Soc. Mass Spectrom. 1994, 4, 316-323, and references therein.

3. Cargile, B. J.; Bundy, J. L.; Stephenson, J. L. Potential for false positive identifications from large databases through tandem mass spectrometry. J. Proteome Res. 2004, 5, 1082-1085.

4. Vessman, J.; Stefan, R. I.; Van Staden, J. F.; Danzer, K.; Lindner, W.; Burns, D. T.; Fajgelj, A.; Muller, H. Selectivity in Analytical Chemistry. Pure Appl. Chem. 2001, 73, 1381-1386.

5. Van Ginkel, L. A.; Stephany, R. W. Experimental chemometrics, an alternative way for estimating overall reliability; Proceedings of the Euroresidues II Conference on Residues of Veterinary Drugs in Food Haagsma, N., Ed.; Veldhoven, Netherlands, May, 1993; pp 303-307.

6. Sphon, J. A. Use of mass spectrometry for confirmation of animal drug residues. J. Assoc. Off. Anal. Chem. 1978, 61, 1247-1252.

7. Baldwin, R.; Bethem, R. A.; Boyd, R.; Budde, W. L.; Cairns, T.; Gibbons, R. D.; Henion, J. D.; Kaiser, M. A.; Lewis, D. L.; Matusik, J. E.; Sphon, J. A.; Stephany, R. W.; Trubey, R. K. Report on 1996 ASMS Fall Workshop: Limits to confirmation, quantitation, and detection. J. Am. Soc. Mass Spectrom. 1997, 8, 1180-1190.

8. Webb, K., Sargent, M. The reliability of mass spectrometry for identification purposes. VAM Bulletin of the Laboratory of the Government Chemist: Teddington, UK; 2000, 22, 12-14

9. Bristow, A. W. T.; Webb, W. S.; Lubben, A. T.; Halket, J. Reproducible product-ion tandem mass spectra on various liquid chromatography/ mass spectrometry instruments for the development of spectral libraries. Rapid Commun. Mass Spectrom. 2004, 18, 1447-1454.

10. Hough, J. M.; Haney, C. A.; Voyksner, R. D.; Bereman, R. D. Evaluation of electrospray transport CID for the generation of searchable libraries. Anal. Chem. 2000, 72, 2265-2270.

11. Josephs, J. L.; Sanders, M. Creation and comparison of MS/MS spectral libraries using quadrupole ion trap and triple-quadrupole mass spectrometers. Rapid Commun. Mass Spectrom. 2004, 18, 743-759.

12. FDA Center for Veterinary Medicine Guidance Document no. 118; Mass Spectrometry for Confirmation of the Identity of Animal Drug Residues, 2003; http:/ /www.fda.gov/cvm/guidance/guide118.pdf

13. Commission Decision Implementing Council Directive 96/23/EC concerning the performance of analytical methods and the interpretation of results. Official J. Eur. Commun. 2002, 221, 8-36. 
14. Bethem, R.; Boison, J.; Gale, J.; Heller, D.; Lehotay, S.; Loo, J.; Musser, S.; Price, P.; Stein, S. Establishing the fitness for purpose of mass spectrometric methods. J. Am. Soc. Mass Spectrom. 2003, 14, 528-541.

15. NIST/EPA/NIH Mass Spectral Library. NIST Standard Reference Database 1A, 2002, NIST, Gaithersburg, MD.

16. Derived from code available through http://chemdata.nist.gov, contact author for details (steve.stein@nist.gov).

17. Stein, S. E. An integrated method for spectrum extraction and com- pound identification from gas chromatography/mass spectrometry data. J. Am. Soc. Mass Spectrom. 1999, 10, 770-781.

18. Pesyna G. M.; Venkataraghavan R.; Dayringer H. E.; McLafferty F. W. Probability based matching system using a large collection of reference mass spectra. Anal. Chem. 1976, 48, 1362-1368.

19. Wan K. X.; Vidavsky, I.; Gross, M. L. Comparing similar spectra: From similarity index to spectral contrast angle. J. Am. Soc. Mass Spectrom. 2002, 13, 85-88. 